\title{
A novel AXIN2 mutation and perinatal risk factors contribute to sagittal craniosynostosis: evidence from a monochorionic diamniotic twin family
}

$\operatorname{Jin} \mathrm{Xu}$

Nanjing Medical University

Qing Yan

Children's Hospital of Nanjing Medical University

Chengcheng Song

Fudan University School of Life Sciences

Jingjia Liang

Nanjing Medical University

Liang Zhao

First Affiliated Hospital of Nanjing medical University

Xin Zhang

Nanjing Medical University

Zhenkun Weng

Nanjing Medical University

Cheng Xu

Nanjing Medical University

Qian Liu

Nanjing Medical University

Shuqin Xu

Nanjing Medical University

Lu Pang

Nanjing Medical University

Liye Zhang

Nanjing Medical University

Yuan Sun

Nanjing Medical University

Gang Wang

Children's Hospital of Nanjing Medical University

Aihua Gu ( $\nabla$ aihuagu@njmu.edu.cn )

Nanjing Medical University https://orcid.org/0000-0001-7576-9998 
Research

Keywords: Sagittal craniosynostosis, AXIN2, perinatal risk factors, mutation, monochorionic diamniotic twin

Posted Date: August 17th, 2020

DOI: https://doi.org/10.21203/rs.3.rs-57248/v1

License: (c) (i) This work is licensed under a Creative Commons Attribution 4.0 International License.

Read Full License 


\section{Abstract}

Background: Craniosynostosis, the premature fusion of one or more cranial sutures, affects approximately 1 in every 2000-2500 live births. The etiology of sagittal craniosynostosis, the most prevalent form of isolated craniosynostosis, involves interplay between genetic and environmental insults, such as perinatal risk factors. However, the detailed information still remains largely unknown.

Methods: The proband, a female monochorionic twin diagnosed with sagittal craniosynostosis, as well as her healthy twin sister and parents, were enrolled in our study. The obstetric medical records were retrospectively reviewed. Genetic cause was investigated by whole exome sequencing (WES) and further confirmed by Sanger sequencing.

Results: We identified a novel heterozygous mutation of AXIN2 (c.1181G >A) in monochorionic twins and their father. However, only the proband presented sagittal craniosynostosis. This mutation results in the replacement of Arg at residue 394 by His (p.R394H). Arg 394 is located at the GSK3 $\beta$ binding domain of the AXIN2 protein, which is highly conserved across species. The obstetric medical records revealed that the proband had additional persistent breech presentation and intrauterine growth restriction, except for the perinatal risk factors shared by the twins.

Conclusions: Based on the role of AXIN2 in craniosynostosis development and deleterious prediction in silico of AXIN2 (c.1181G>A: p.R394H), we speculate that this particular mutation confers susceptibility to sagittal craniosynostosis, while extra environmental insults are also involved in the pathogenesis of this case. Our findings provide a new evidence for the gene-environment interplay in understanding pathogenesis of craniosynostosis.

\section{Introduction}

Craniosynostosis (CS), defined as premature fusion of one or more cranial sutures, affects approximately 1 in every 2000-2500 live births[1]. It usually involves as an isolated condition (non-syndromic craniosynostosis, NCS), but may also be associated with other malformations as part of complex syndromes (syndromic craniosynostosis)[2]. Sagittal craniosynostosis is the most prevalent form of NCS, which accounts for $40-58 \%$ of all NCS cases[3]. Sagittal suture premature closure restricts the widen of the skull, thus causing the scaphocephaly deformity and other adverse neurologic outcomes[4].

The pathoetiology of NCS, involving interplay between genetic and environmental factors, is still not well defined[5-8]. FGF (fibroblast growth factor), BMP (bone morphogenic protein), Wnt (wingless-type integration sites) pathways are the major regulators in suture biology $[9,10]$. Mutations of pivotal components in these signaling pathways, including FGFR2 (fibroblast growth factor receptor 2), TWIST1 (twist, drosophila, homolog of 1), AXIN2 (axis inhibitor 2), have been identified the origins of craniosynostosis[1, 9]. Non-genetic risk factors, such as intrauterine constraint, twin gestation, low birth weight, malnutrition, premature delivery, maternal thyroid disorders, gestational diabetes, virus infectious, can either cause or exacerbate craniosynostosis[7, 11-14]. Although several findings demonstrate the 
interactions between genetic and environmental influences that contribute to prematurely fused sutures $[5$, 15], more evidence are still needed to delineate the institutional patterns.

Monochorionic (MC) twins, sharing almost the same genome, offer a unique opportunity to study the gene/environment interactions, for the healthy twin as an ideal control. Discordant phenotypes and lack of $100 \%$ concordance between MC twins emphasize the interplay between genetic and environmental influences in the etiology of the disease[16].

In our study, we reported a female monochorionic twin, who was diagnosed with sagittal craniosynostosis, carried a novel heterozygous mutation of $A X I N 2$ (c.1181G>A: p.R394H). This mutation also exists in the patient's father and her monochorionic sister; however, neither of whom present craniosynostosis. Intriguingly, the obstetric history documented that the proband had a persistent breech presentation and intrauterine growth restriction. We speculate that this particular AXIN2 mutation confers susceptibility, and additional environmental insults eventually trigger the sagittal craniosynostosis to occur.

\section{Materials And Methods}

\section{Clinic examination}

All participants signed the informed consent and received physical examination in the craniofacial clinic.

\section{Whole exome sequencing and data analysis}

Genomic DNA, extracted from peripheral blood samples (I-1, I-2, II-1 and II-2) and skull periosteum tissue (II-1), was subjected to whole-exome sequencing (WES) on the platform of Genergy Biotechnology, Shanghai, China. Raw reads were aligned to the human genome reference assembly (GRCh37/hg19) using the Burrows-Wheeler Aligner[17]. The Picard software was employed to remove PCR duplicates and evaluate the quality of variants. DNA variants was called and analyzed using the Genome Analysis Toolkit[18]. The variants with read depths less than $4 \times$ were filtered out. All variants were further annotated using the ANNOVAR tool with the information of the Exome Aggregation Consortium (ExAC) Browser, Sorting Intolerant From Tolerant (SIFT), Polymorphism Phenotyping v2 (PolyPhen-2), MutationTaster, Online Mendelian Inheritance in Man (OMIM), Gene Ontology and KEGG Pathway databases[19-25]. The workflow of our genetic analysis is shown in Fig. 1.

\section{Sanger sequencing}

The AXIN2 mutation was validated by Sanger sequencing using the following primers: Forward: $5^{\prime}-$ CGCACACCCTAACGCACCCCAT-3' and Reverse: 5'-ACCGCCCACCTAGCCTGCTGAA-3', which was performed by Tsingke, China. The data were then visualized and analyzed using FinchTV (Geospiza) software.

\section{Conservation analysis}


Multiple-species amino acid sequences were obtained from National Center for Biotechnology Information (NCBI), and analyzed by WebLogo (Version 2.8.2, http://weblogo.berkeley.edu)[26].

\section{Structural analysis}

Three-dimensional models of the wild-type and mutant site (p.R394H) of AXIN2 protein were designed using the I-TASSER[27]. The models were manipulated and visualized using the PyMOL software (PyMOL Molecular Graphics System, DeLano Scientific, San Carlos, CA).

\section{Results}

\section{Clinical information}

The pedigree came from Wuhan, Hubei Province, China. The female proband (II-1), the elder monochorionic diamniotic (MCDA) twin (Fig. 2a-c and Addition file: Fig. S1-3), was diagnosed with sagittal craniosynostosis at the age of 9 months in the Department of Neurosurgery, Children's Hospital of Nanjing Medical University. She was born to non-consanguineous parents without family history of craniosynostosis. Her mother, a 30-year-old Chinese female, conceived MCDA twins by frozen embryo transfer (FET) (Addition file: Fig. S1-3). Her father was 36-year-old at that time. The 26 days after FET, four-dimensional ultrasound scan confirmed two embryos were inside a gestational sac (approximately $22 \mathrm{~mm} \times 13 \mathrm{~mm}$ ); one embryo was $2.8 \mathrm{~mm}$ in length and the fetal heart rate was 107 per minute, the other embryo was $3.3 \mathrm{~mm}$ in length and the fetal heart rate was 118 per minute (Addition file: Fig. S1).

At 13 weeks of gestation, IgG of cytomegalovirus and herpes simplex virus were detected in the mother's serum by the toxoplasmosis, rubella, cytomegalovirus and herpes simplex (TORCH) test (Addition file: Table S1). At 14 weeks of gestations, maternal dietary assessment indicated that the mother had inadequate intakes of energy, protein, fat, vitamins and several other minerals (Addition file: Table S2); and non-invasive prenatal testing demonstrated low risks for Trisomy 13, 18 and 21 (Addition file: Table S3). At 17 weeks of gestations, elevated levels of thyroid-stimulating hormone (TSH) and urinary iodine were detected (Addition file: Table S4). At 27 weeks of gestations, the mother was diagnosed with gestational diabetes mellitus (fasting blood glucose level, $7.38 \mathrm{mmol} / \mathrm{L}$; postprandial one-hour blood glucose level, $15.30 \mathrm{mmol} / \mathrm{L}$; postprandial two-hour blood glucose level, $11.03 \mathrm{mmol} / \mathrm{L}$ ).

At 28 weeks of gestations, the proband (II-1) and her twin younger sister (II-2) were born via spontaneous vaginal delivery (Fig. 2a, b). The proband (II-1), who had been in persistent breech position on the left side of mother's uterus (Fig. 2b), was first recorded by ultrasound scan at 23.5 weeks of gestation; her birth weight was $880 \mathrm{~g}$ (50-90 centile[28]). The younger sister (II-2), who had been in cephalic position on the right side of mother's uterus (Fig. 2b), weighted $990 \mathrm{~g}$ (90-97 centile[28]) at birth. Only the proband presented the typical appearance of sagittal craniosynostosis (Fig. 2C). While no signs of craniofacial deformity were found in her parents (I-1, I-2) and sister (II-2) (Fig. 2C). Cranial index, which represents the ratio of maximum cranial width to maximum cranial length[29], is decreased in the head shape of sagittal craniosynostosis. In our case, the cranial index of the proband and her sister are $70.9 \%, 83.2 \%$, 
respectively. Taken together, the clinical findings indicate that the proband, who developed sagittal craniosynostosis, had additional persistent breech presentation and intrauterine growth restriction, except for the perinatal risk factors shared by the twins.

\section{Mutation analysis}

The whole-exome sequencing (WES) was applied to uncover the potential genetic etiology leading to sagittal craniosynostosis in our case. Due to the low incidence rate of sagittal craniosynostosis[3], we focused on private and/or rare (minor allele frequency, MAF $<0.01$ ) variants on exons or splicing sites (Fig. 1). However, none of candidate germline or somatic mutations were proband-specific. As geneenvironment interactions have been revealed in the pathogenesis of craniosynostosis [5, 15], we wonder whether the adverse intrauterine exposures (environment) triggered the susceptible individual (II-1) to develop sagittal craniosynostosis. Based on this hypothesis, we re-analyzed our sequencing data and identified a heterozygous missense mutation of $A X I N 2$ (c.1181G>A, p.R394H) in the leukocytes of subjects I-1, II-1 and II-2, and skull periosteum tissue of subject II-1. The findings were further validated by Sanger sequencing (Fig. 3a-b). The nucleotide sequence showed a heterozygous $G$ to $A$ transition at nucleotide 1181 (c.1181 G>A) of the coding sequence, which resulted in the replacement of Arg at residue 394 by His of AXIN2 protein.

\section{Conservation analysis}

Arg394, located at the GSK3 $\beta$ binding domain (amino acid 327 to 413 according to the UniProt Consortium) of the AXIN2 protein (Fig. 3c)[30], is conserved across species (Fig. 3d, e) and was predicted to be potentially deleterious by in silico analysis (Table 1 ).

\section{Structural analysis}

I-TASSER structural analysis predicted that the p. R394H substitution may affect the spatial structure of the GSK3 $\beta$ binding domain in AXIN2 protein (Fig. $4 \mathrm{a}, \mathrm{b}$ ). Taken together, our results indicate that AXIN2 (c.1181G>A, p.R394H) mutation may confer deleterious of sagittal craniosynostosis.

\section{Discussion}

Craniosynostosis, a highly heterogeneous disease, is caused by genetic mutations, adverse exposures as well as their interactions. The study of gene/environment interactions provides the foundation for clarifying the pathogenesis of craniosynostosis, identifying susceptibility individuals, discerning modifiable environmental risk factors and establishing effective strategies for prevention and early 
diagnosis. In our study, we demonstrated that a novel heterozygous AXIN2 (c.1181G>A: p.R394H) mutation was presented in three members of the family, including the monochorionic twins and their father. However, only the female proband with an additional environmental insult, that is, persistent breech presentation and intrauterine growth restriction in our case, developed overt craniosynostosis. We assume that this AXIN2 mutation predisposes to sagittal craniosynostosis but needs extra environmental insults to initiate the disease.

It has been well accepted that $A X I N 2$ is essential for normal calvarial morphogenesis by directly targeting $\beta$-catenin, orchestrating the crosstalk of Wnt, BMP, FGF signaling pathways and maintaining suture cell stemness[31-33]. Deletion or mutation of AXIN2 attribute to premature suture closure and craniosynostosis in humans and mice[6,32]. Moreover, phenotype data available in International Mouse Phenotyping Consortium (IMPC, https://www.mousephenotype.org/) show that all the female and male homozygous AXIN2 knockout mice present calvarial malformation. For heterozygous AXIN2 deletion mice, only 2/7 females develop an abnormal head shape; however, the males are not observed any noticeable distinctions, which indicates the incomplete penetrance of $A X I N 2$ mutations. In this study, an AXIN2 heterozygous missense mutation (c.1181G >A) was identified in peripheral blood samples of subjects I-1, II-1 and II-2 (Fig. 1a), which indicates that the proband inherits the mutation from her father. The function of $A X I N 2$ (c.1181G >A) has not been reported according to the lasted updates in ClinVar (Record last updated Dec 17, 2019, https://www.ncbi.nlm.nih.gov/clinvar/variation/234485/). Our findings indicate that this mutation is conserved across species and was likely to be deleterious by in silico prediction tools; however, only the proband suffered sagittal craniosynostosis. We assumed that the reason of phenotypic segregation in our case is probably because AXIN2 (c.1181G>A) mutation possesses incomplete penetrance, thus making it insufficiency to trigger the disease alone. However, further clinical observations and animal studies are still needed to validate our hypothesis.

Prenatal risk factors, such as maternal thyroid disorders, gestational diabetes, drug use, malnutrition, virus infectious, intrauterine constraint, twin gestation, premature delivery, are associated with craniosynostosis and likely increase the susceptibility in an already genetically predisposed infant [7, 1114]. Monochorionic (MC) twins provide exceptional chances to decipher the interplay among genetic and environment influences in the pathogenesis of premature suture fusion[34]. In our study, the mother of monochorionic twins suffered the majority of risk factors prenatally, however, only the infant with breech presentation and intrauterine growth restriction experienced sagittal craniosynostosis. Our findings corroborate another well-established gene-environment interaction model of NCS, which substantiates almost the same environmental insults ultimately determining phenotype[15]. In all, we highlight that the intrauterine growth restriction and breech position, deserves particularly attention in predicting sagittal craniosynostosis occurrence.

However, our gene-environment interaction fashion was observed in the context of $A X I N 2$ (c.1181G>A) mutation with small sample size and unforeseen bias, which is unlikely to provide accurate assessments for all types NCS. And we also cannot evaluate the epigenetic role in pathogenesis of this case. Further epidemiological and experimental studies are encouraged to give a more unambiguous pattern. 


\section{Conclusion}

In summary, we described a monochorionic twin with AXIN2 (c.1181G>A) mutation suffered sagittal craniosynostosis in the insult of prenatal risk factors (intrauterine growth restriction and breech position). Our findings provide a new evidence for the gene-environment interplay in etiology of NCS, which will be informative in the molecular diagnosis and genetic counselling in clinic.

\section{Abbreviations}

WES: Whole exome sequencing; CS: Craniosynostosis; NCS: Non-syndromic craniosynostosis; FGF: Fibroblast growth factor; FGFR2: Fibroblast growth factor receptor 2; BMP: Bone morphogenic protein; TWIST1: Twist, drosophila, homolog of 1; AXIN2: Axis inhibitor 2; MC twins: Monochorionic (mc) twins; ExAC: Exome aggregation consortium; SIFT: Sorting intolerant from tolerant; PolyPhen-2: Polymorphism phenotyping v2; OMIM: Online mendelian inheritance in man; NCBI: National center for biotechnology information; PyMOL: Molecular graphics system, delano scientific; MCDA: Monochorionic diamniotic; FET: Frozen embryo transfer; MAF: Minor allele frequency

\section{Declarations}

Acknowledgments

We thank Xi Chen for her kind help in drawing the schematic diagram of fetal position.

Authors' contributions

AHG, GW, JX designed the study. GW, QY, LZ provided patients' data and performed clinical assessments. JX, CCS, JJL, ZKW, CX, QL, SQX, LYZ, YS conducted the experiments. JX, QY, CCS, JJL, XZ, ZKW analysed the data. JX, QY, CCS, JJL wrote the manuscript. All authors critically revised the manuscript for important intellectual content. AHG, GW supervised the study.

\section{Fundings}

This work was financially supported by National Key Research and Development Program of China (2019YFA0802701, 2018YFC1004203), the National Science Fund for Outstanding Young Scholars (81722040), the National Natural Science Foundation of China (91839102, 81703261), the Natural Science Foundation of Jiangsu Province (BK20171060), the Natural Science Foundation of the Jiangsu Higher Education Institutions of China (17KJB330002), the Jiangsu Students' Platform for Innovation and Entrepreneurship Training Program (201810312036Y). 
Availability of data and materials

The data of whole-exome sequencing are available upon request.

Ethical Approval and Consent to participate

This research was carried out according to the principles of the Declaration of Helsinki and was approved by the Nanjing Medical University Ethics Committee. All participants have signed informed consent forms.

Consent for publication

Not applicable.

Competing interests

The authors declare that they have no competing interests.

\section{References}

1. Timberlake AT, Persing JA: Genetics of Nonsyndromic Craniosynostosis. Plast Reconstr Surg 2018, 141(6):1508-1516.

2. Paumard-Hernandez B, Berges-Soria J, Barroso E, Rivera-Pedroza Cl, Perez-Carrizosa V, Benito-Sanz S et al: Expanding the mutation spectrum in 182 Spanish probands with craniosynostosis: identification and characterization of novel TCF12 variants. European journal of human genetics : EJHG 2015, 23(7):907-914.

3. Justice CM, Yagnik G, Kim Y, Peter I, Jabs EW, Erazo M et al: A genome-wide association study identifies susceptibility loci for nonsyndromic sagittal craniosynostosis near BMP2 and within BBS9. Nat Genet 2012, 44(12):1360-1364.

4. Dempsey RF, Monson LA, Maricevich RS, Truong TA, Olarunnipa S, Lam SK et al: Nonsyndromic Craniosynostosis. Clin Plast Surg 2019, 46(2):123-139.

5. Durham EL, Howie RN, Cray JJ: Gene/environment interactions in craniosynostosis: A brief review. Orthod Craniofac Res 2017, 20 Suppl 1(Suppl 1):8-11. 
6. Yilmaz E, Mihci E, Guzel Nur B, Alper OM: A novel AXIN2 gene mutation in sagittal synostosis. Am J Med Genet A 2018, 176(9):1976-1980.

7. Sergesketter AR, Elsamadicy AA, Lubkin DT, Krucoff KB, Krucoff MO, Muh CR: Characterization of Perinatal Risk Factors and Complications Associated With Nonsyndromic Craniosynostosis. J Craniofac Surg 2019, 30(2):334-338.

8. Miller KA, Twigg SR, McGowan SJ, Phipps JM, Fenwick AL, Johnson D et al: Diagnostic value of exome and whole genome sequencing in craniosynostosis. J Med Genet 2017, 54(4):260-268.

9. Timberlake AT, Furey CG, Choi J, Nelson-Williams C, Yale Center for Genome A, Loring E et al: De novo mutations in inhibitors of Wnt, BMP, and Ras/ERK signaling pathways in non-syndromic midline craniosynostosis. Proc Natl Acad Sci U S A 2017, 114(35):E7341-E7347.

10. Katsianou MA, Adamopoulos C, Vastardis H, Basdra EK: Signaling mechanisms implicated in cranial sutures pathophysiology: Craniosynostosis. BBA Clin 2016, 6:165-176.

11. Sanchez-Lara PA, Carmichael SL, Graham JM, Jr., Lammer EJ, Shaw GM, Ma C et al: Fetal constraint as a potential risk factor for craniosynostosis. American journal of medical genetics Part A 2010, 152A(2):394-400.

12. Lee HQ, Hutson JM, Wray AC, Lo PA, Chong DK, Holmes AD et al: Changing epidemiology of nonsyndromic craniosynostosis and revisiting the risk factors. The Journal of craniofacial surgery 2012, 23(5):1245-1251.

13. James AW, Levi B, Xu Y, Carre AL, Longaker MT: Retinoic acid enhances osteogenesis in cranial suture-derived mesenchymal cells: potential mechanisms of retinoid-induced craniosynostosis. Plastic and reconstructive surgery 2010, 125(5):1352-1361.

14. Carmichael SL, Rasmussen SA, Lammer EJ, Ma C, Shaw GM, National Birth Defects Prevention S: Craniosynostosis and nutrient intake during pregnancy. Birth Defects Res A Clin Mol Teratol 2010, 88(12):1032-1039.

15. Johnson D, Wall SA, Mann S, Wilkie AO: A novel mutation, Ala315Ser, in FGFR2: a gene-environment interaction leading to craniosynostosis? European journal of human genetics : EJHG 2000, 8(8):571577.

16. Derom C, Thiery E, Rutten BPF, Peeters H, Gielen M, Bijnens E et al: The East Flanders Prospective Twin Survey (EFPTS): 55 Years Later. Twin Res Hum Genet 2019:1-6.

17. Li H, Durbin R: Fast and accurate long-read alignment with Burrows-Wheeler transform. Bioinformatics (Oxford, England) 2010, 26(5):589-595.

18. McKenna A, Hanna M, Banks E, Sivachenko A, Cibulskis K, Kernytsky A et al: The Genome Analysis Toolkit: a MapReduce framework for analyzing next-generation DNA sequencing data. Genome research 2010, 20(9):1297-1303.

19. Wang K, Li M, Hakonarson H: ANNOVAR: functional annotation of genetic variants from highthroughput sequencing data. Nucleic acids research 2010, 38(16):e164.

20. Lek M, Karczewski KJ, Minikel EV, Samocha KE, Banks E, Fennell T et al: Analysis of protein-coding genetic variation in 60,706 humans. Nature 2016, 536(7616):285-291. 
21. Kumar P, Henikoff S, Ng PC: Predicting the effects of coding non-synonymous variants on protein function using the SIFT algorithm. Nature protocols 2009, 4(7):1073-1081.

22. Adzhubei IA, Schmidt S, Peshkin L, Ramensky VE, Gerasimova A, Bork $P$ et al: A method and server for predicting damaging missense mutations. Nature methods 2010, 7(4):248-249.

23. Schwarz JM, Cooper DN, Schuelke M, Seelow D: MutationTaster2: mutation prediction for the deepsequencing age. Nature methods 2014, 11(4):361-362.

24. Ashburner M, Ball CA, Blake JA, Botstein D, Butler H, Cherry JM et al: Gene ontology: tool for the unification of biology. The Gene Ontology Consortium. Nature genetics 2000, 25(1):25-29.

25. Kanehisa M, Furumichi M, Tanabe M, Sato Y, Morishima K: KEGG: new perspectives on genomes, pathways, diseases and drugs. Nucleic acids research 2017, 45(D1):D353-d361.

26. Crooks GE, Hon G, Chandonia JM, Brenner SE: WebLogo: a sequence logo generator. Genome Res 2004, 14(6):1188-1190.

27. Roy A, Kucukural A, Zhang Y: I-TASSER: a unified platform for automated protein structure and function prediction. Nat Protoc 2010, 5(4):725-738.

28. Villar J, Giuliani F, Bhutta ZA, Bertino E, Ohuma EO, Ismail LC et al: Postnatal growth standards for preterm infants: the Preterm Postnatal Follow-up Study of the INTERGROWTH-21(st) Project. Lancet Glob Health 2015, 3(11):e681-691.

29. Bendon CL, Sheerin FB, Wall SA, Johnson D: The relationship between scaphocephaly at the skull vault and skull base in sagittal synostosis. J Craniomaxillofac Surg 2014, 42(3):245-249.

30. Ren J, Wen L, Gao X, Jin C, Xue Y, Yao X: DOG 1.0: illustrator of protein domain structures. Cell Res 2009, 19(2):271-273.

31. Liu B, Yu HM, Hsu W: Craniosynostosis caused by Axin2 deficiency is mediated through distinct functions of beta-catenin in proliferation and differentiation. Dev Biol 2007, 301(1):298-308.

32. Yu H-MI, Jerchow B, Sheu T-J, Liu B, Costantini F, Puzas JE et al: The role of Axin2 in calvarial morphogenesis and craniosynostosis. Development 2005, 132(8):1995-2005.

33. Maruyama T, Jeong J, Sheu T-J, Hsu W: Stem cells of the suture mesenchyme in craniofacial bone development, repair and regeneration. Nat Commun 2016, 7:10526-10526.

34. Lakin GE, Sinkin JC, Chen R, Koltz PF, Girotto JA: Genetic and epigenetic influences of twins on the pathogenesis of craniosynostosis: a meta-analysis. Plastic and reconstructive surgery 2012, 129(4):945-954.

\section{Table}

Table 1: In silico predictions of c.1181G>A (p.R394H) mutation on AXIN2 function 
AXIN2 c.1181G>A

Allele Frequency in Human Populations

Global in 1000 Genomes Project Not recorded

Global in ExAC $\quad 0.0006369$

Global in gnomAD $\quad 0.000127397$

Conservation

$\begin{array}{ll}\text { PhastCons } & 1 \\ \text { Phylop } & 5.36\end{array}$

Functional Prediction

\begin{tabular}{ll} 
SIFT & Deleterious \\
\hline PolyPhen-2 & Possibly damaging \\
\hline MutationTaster & Disease causing
\end{tabular}

\section{Figures}




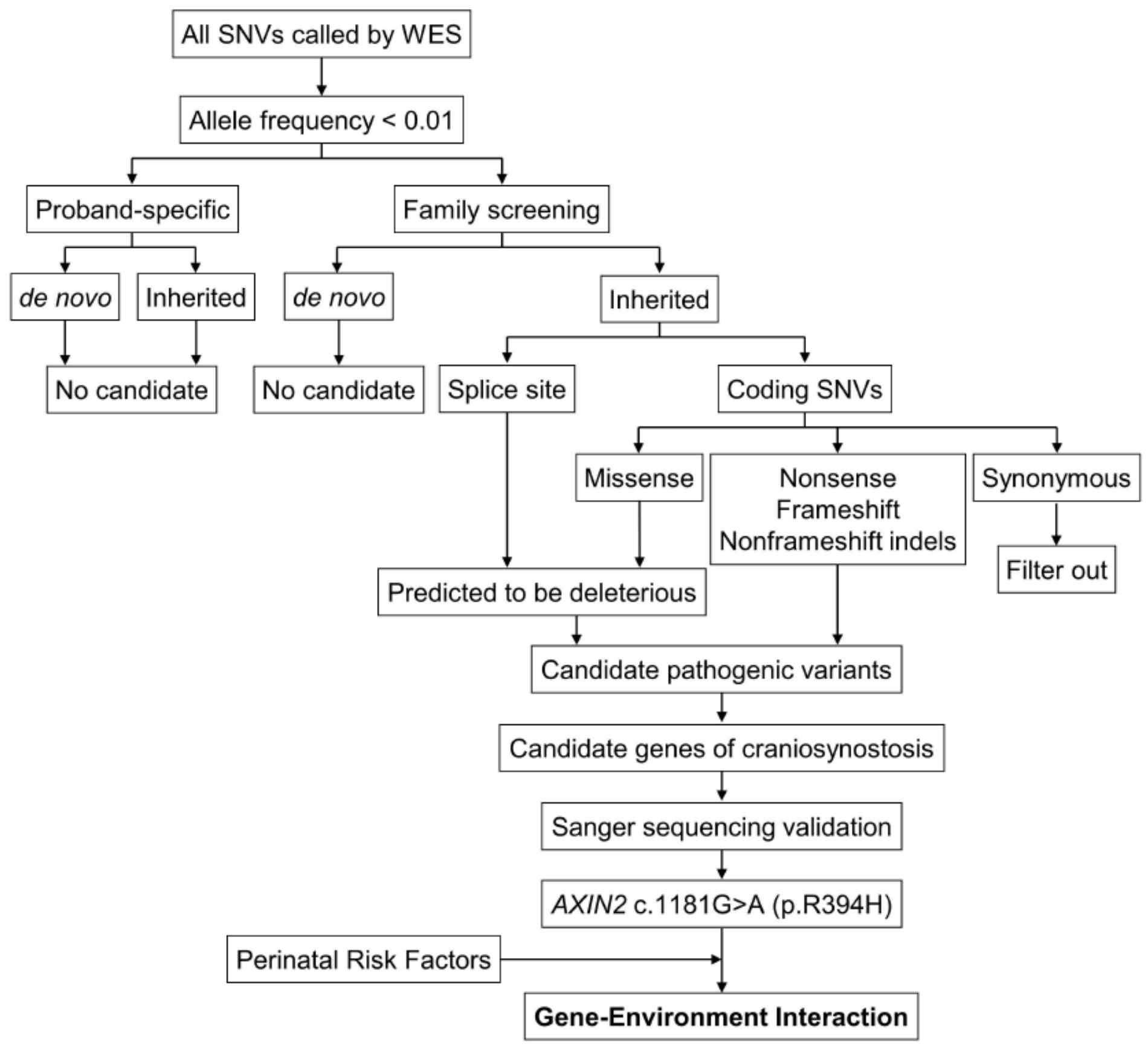

Figure 1

Workflow for the genetic analyses using WES and Sanger sequencing in pedigree diagnosed with sagittal craniosynostosis. 
a

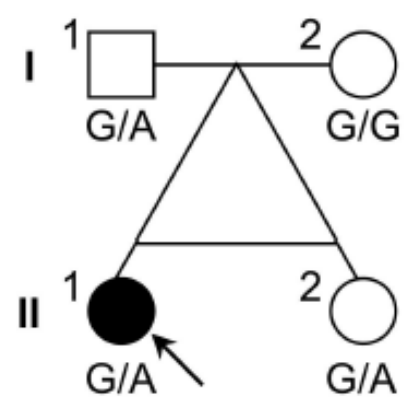

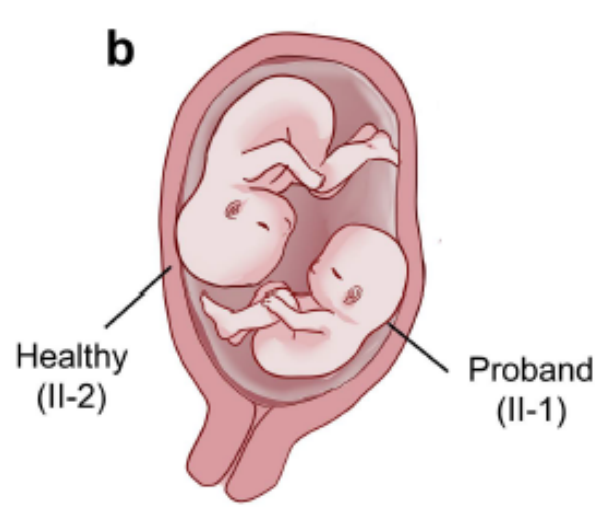

c

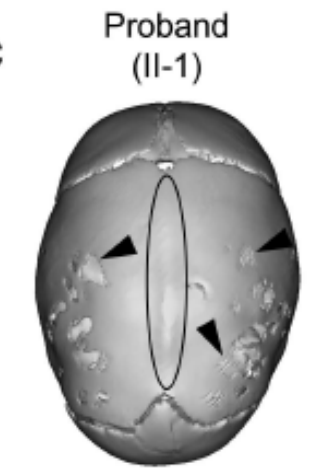

Cranial index $=70.9 \%$ Cranial index $=83.2 \%$

Figure 2

Clinical characteristics of the proband with sagittal craniosynostosis. (a) The pedigree of the family. The proband is marked with an upward arrow. Open squares/circles denote unaffected individuals; squares denote males and circles denote females. (b) Schematic diagram of the fetal position before delivery. The proband (II-1) was in breech position, while her healthy sister (II-2) was in cephalic position. (c) the computerized tomographic (CT) scan revealed fused sagittal suture (solid circle) and digital impressions (arrowheads) in the proband (left panel). Cranial index of the proband is $70.9 \%$. The proband's sister was also received CT examination under the request of the parents for ruling out craniosynostosis. Her sagittal suture remains patent (dotted circle) and cranial index is $83.2 \%$ (right panel). 
a

AXIN2 c.1181G (wild type)

TGGAGGAGCGCCTGCAGCA

Healthy

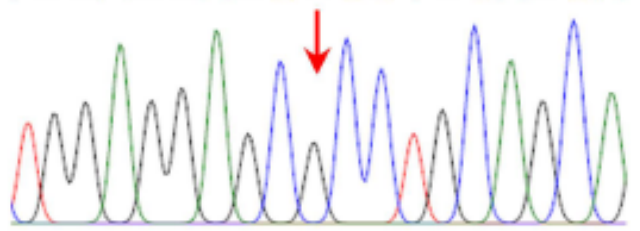

b

AXIN2 c.1181A (mutation)

TGGAGGAGCACCTGCAGCA

Patient

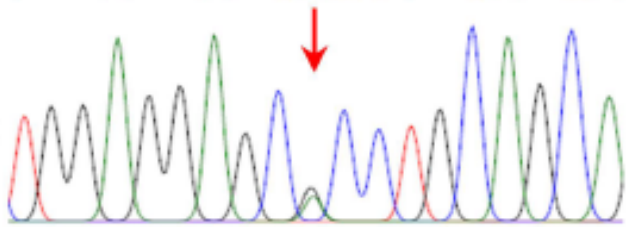

C TNKS_binding AXIN2 p.R394H

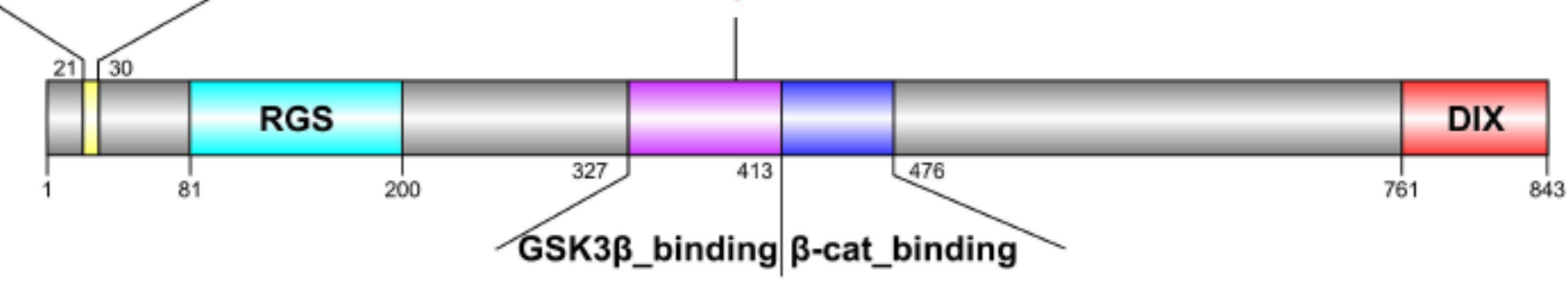

d

Homo sapiens

Pan troglodytes

AXIN2 p.R394H

Canis lupus familiaris

Bos Taurus

Mus musculus

Rattus norvegicus

Gallus gallus

e

Danio rerio

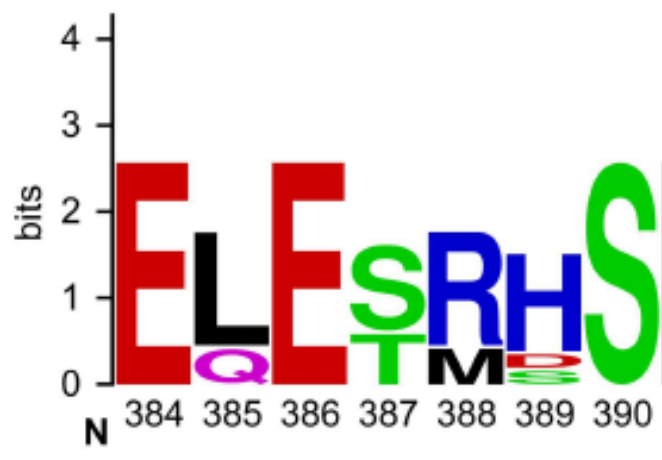

ELESRHSLEERLQQIREDEER

ELESRHSLEERLQQIREDEEK

ELESRHSLE ERLQQI REDEEK

ELETRHSLEERLQQI QEDEEK ELESRHSLEERLQQ I REDEEK ELESRHSLE ERLQQI REDEEK E Q E T M D S L E ERL Q Q I KEDEEK E Q E T M S S L E RL Q Q I Q E E E R

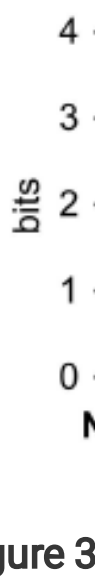

Sequence, conservation analyses of c.1181G >A (p.R394H). (a, b) A heterozygous mutation, c.1181G $>A$, was found in the proband as well as her father and sister (b), but not in her mother (a). (c) Schematic diagram depicts the protein structure of AXIN2. The 394th amino acid position, which resides in the GSK3ß_binding domain, is mutated R394H. (d) Evolutionary conservation analysis revealed that the Arg394 site is conserved from zebrafish to humans. (e) WebLogo analysis showed that the Arg394 site 
was relatively conserved. TNKS_binding: Tankyrase binding N-terminal segment of axin; RGS: Regulator of G protein signaling; DIX: Disheveled and AXIN interacting.

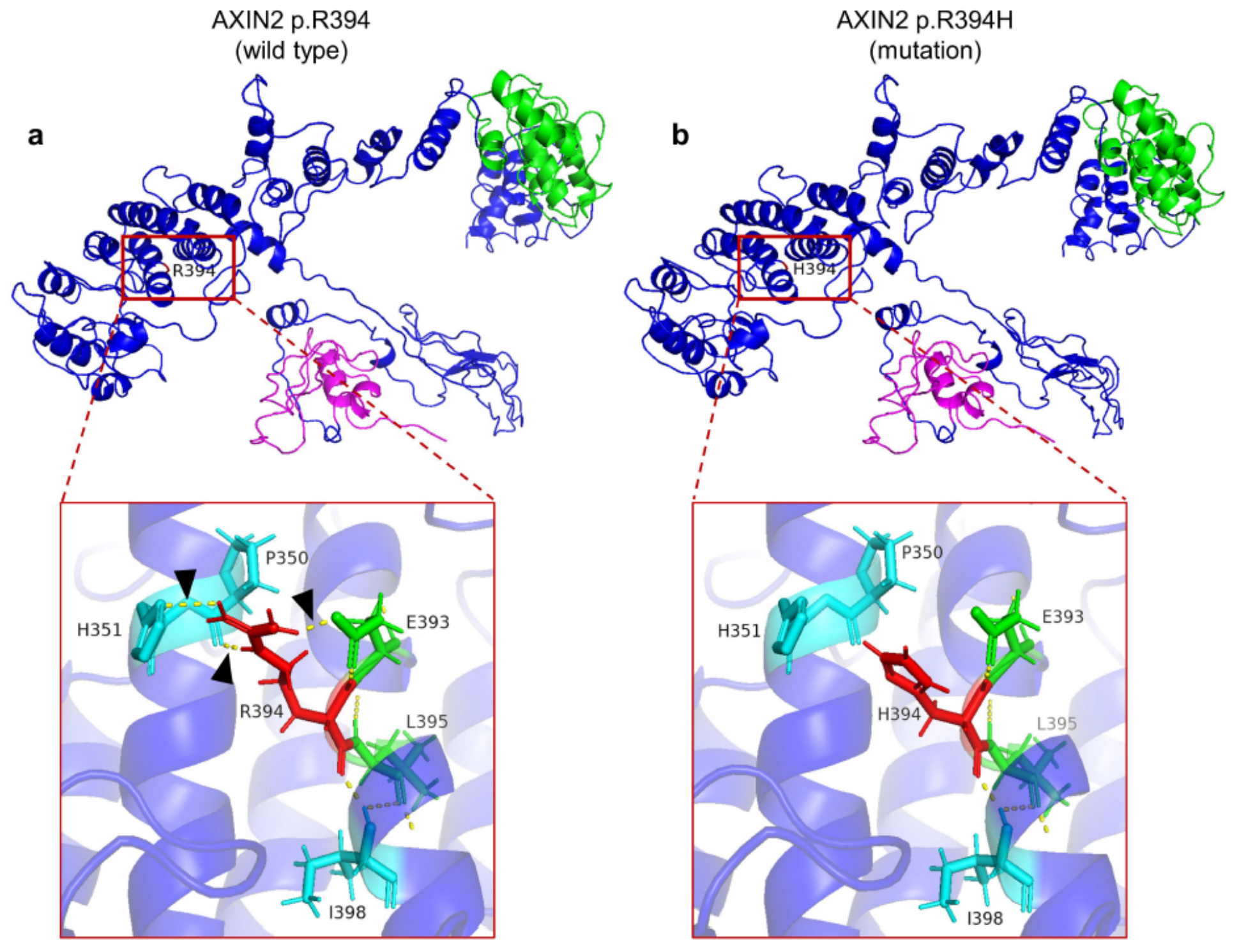

Figure 4

Spatial structure of the GSK3 $\beta$ binding domain of the AXIN2 protein. (a) Location of the Arg394 residues within the GSK3 $\beta$ binding domain of AXIN2. Arrowheads indicate the hydrogen bond in the domain. (b) Location of the His 394 residues within the GSK3 $\beta$ binding domain of AXIN2.

\section{Supplementary Files}

This is a list of supplementary files associated with this preprint. Click to download.

- Additionfile.docx 